\title{
An Eleven-Year Review of Congenital Pulmonary Lesions in a Referral Children's Hospital in Tehran
}

\author{
Lobat Shahkar,, Sara Sanii,, ${ }^{2,}$ Habibe Nejad Biglari, ${ }^{3}$ Elham Pourbakhtyaran, ${ }^{2}$ Seyyed Ahmad \\ Tabatabai, ${ }^{1}$ and Seyyed Hossein Fakhraee ${ }^{2}$ \\ ${ }^{1}$ Pulmonology Department, Shahid Beheshti University of Medical Sciences, Tehran, Iran \\ ${ }^{2}$ Neonatal Health Research Center, Shahid Beheshti University of Medical Sciences, Tehran, Iran \\ ${ }^{3}$ Pediatric Neurology Department, Shahid Beheshti University of Medical Sciences, Tehran, Iran \\ "Corresponding author: Sara Sanii, Neonatal Health Research Center, Shahid Beheshti University of Medical Sciences, Tehran, Iran. Tel: +98-9122101653, Fax: +98-2122019321, \\ E-mail: sanii_sara@yahoo.com
}

Received 2015 August 29; Revised 2016 July 27; Accepted 2016 August 07.

\begin{abstract}
Background: Congenital pulmonary lesions may be diagnosed through ultrasonographic screenings or be revealed as causes of respiratory distress in the neonatal period and infancy. Less commonly, they are detected as incidental features.

Objectives: Our study represents the diversity of congenital pulmonary lesions and their characteristics during an 11-year period in a referral teaching children's hospital in Tehran, the capital city of Iran.

Methods: Data from an 11-year period of patients with the final diagnosis of congenital pulmonary lesions in Mofid Children's hospital were reviewed. The data included the prenatal ultrasonographic, postnatal radiographic, and pathologic diagnoses, along with the patient's age, way of presentation, length of hospitalization, and accompanying features and morbidities.

Results: Of 37 cases of congenital pulmonary lesions, 28 cases (75\%) were boys. Thirty-six cases (97.2\%) presented with pure pulmonary signs and symptoms. Of these cases, 16 (43.2\%) were neonates, 17 (46\%) were infants, and 4 (10.8\%) were children. Twentyseven (73\%) patients missed the opportunity for early diagnosis. In order of frequency, cases were diagnosed as congenital lobar emphysema (43.5\%), congenital cystic adenomatoid malformation (32.5\%), pulmonary sequestration (19\%), hybrid lesion (2.5\%), or bronchogenic cyst (2.5\%).

Conclusions: In an analysis of retrospective data of 37 congenital pulmonary lesions, male predominance was obvious, as has been found in previous studies.
\end{abstract}

Keywords: Pulmonary Emphysema, Congenital, Bronchopulmonary Sequestration, Cystic Adenomatoid Malformation of Lung, Congenital

\section{Background}

Congenital pulmonary lesions in children may present diversely in different age groups. Annual incidence of congenital thoracic malformation is $4.4 / 10,000$ (1). A subdivision of these is categorized as cystic lesions (2). There have always been controversies about their origin, nomenclature, pathology, and even therapeutic approaches $(1,2)$. Congenital pulmonary airway malformation (CPAM), previously called congenital cystic adenomatoid malformation (CCAM), with the incidence of $1 / 11,000$ to $1 / 35,000$, is subdivided into five types (1-3). It is known that this lesion has the potential for prenatal regression and that it may retain its primary size or grow and bring about pulmonary hypoplasia and hydrops $(2,3)$. Early diagnosis by gestational routine ultrasonography, with positive predictive value and the sensitivity of ranges between $45 \%$ - 57\% and $70 \%-81 \%$, respectively, is of great value in many ways (4). First, if indicated, by fetal interventions, growth and normal development of pulmonary tissue will be guaranteed. Second, the consequences of a maladapted newborn, such as respiratory distress, long hospitalizations, frequent infectious complications, and malignancy transformation will be prevented. Third, by serial ultrasonographic monitoring, the natural course of the lesions (e.g., regressing, progressing, reaching a plateau, or even disappearing) can be followed.

Bronchopulmonary Sequestrations (BPSs), as nonfunctional pulmonary tissues, comprise up to $14 \%$ to $30 \%$ of congenital lung lesions (1-3). They are classified as extra lobar sequestration (ELS) and intra lobar sequestration (ILS). ILS is often located in the posterior basal segment of the left lower lobe. ELS, in almost $50 \%$ of cases, has 
associated malformations, such as congenital diaphragmatic hernia (1-3). ELS is found more often in the left lower lobe and is more prevalent in males (3). These two groups differ regarding venous drainage; ILS is drained into the pulmonary, but ELS is drained into the systemic (e.g., azygos or portal vein) venous system $(1,3)$. Detection of vascular supply is important for surgical excisional planning. There may be found a prenatal history of polyhydramnios (1-3). Moreover, some of these lesions seem to disappear postnatally. These lesions have a more favorable outcome than CCAMs $(2,3)$. The combination of an aberrant systemic blood supply and an echogenic lung mass in prenatal ultrasonography is pathognomonically in favor of sequestration (2).

Congenital Lobar Emphysema (CLE) is mostly seen in males and affects the left upper lobe (42\%), right middle lobe (35\%), right upper lobe (21\%), and lower lobes (2\%), in sequence of prevalence $(2,3)$. Spontaneous regression in the third trimester is not unusual in CLEs. Asymptomatic lesions, or ones with minimal symptoms, may be followed conservatively, contrary to CCAMs and PSs, which hold the risk of later infection and malignancy transformation. CLE is rarely documented in prenatal ultrasonographies $(2,3)$.

Bronchogenic cysts (BCs) are found in the mediastinum in up to two-thirds of cases, with the remainder found within the lung parenchyma (1-3). Most cases present postnatally, usually with pulmonary infection. Malignant transformation has not been reported in relation to these lesions. Some clinicians choose to manage them conservatively, while others prefer surgery, based on the patient's symptoms or the mass's complications (1-3).

\section{Objectives}

The aim of this study was to demonstrate the diversity of congenital pulmonary masses, define the applied diagnostic modalities of each, and report an analysis of demographic data and patients' various presenting features.

\section{Methods}

This retrospective study was conducted in Mofid children's hospital, as the most crowded referral pediatric surgery center, to provide readers with comprehensive data about each congenital mass group's characteristics and make comparisons with similar studies. All patients included in this study were diagnosed prenatally or postnatally as congenital pulmonary mass, incidentally detected during hospital course as congenital pulmonary mass, or referred for further investigation between the years of 2004 and 2015. After obtaining approval from the ethical committee of Shahid Beheshti University of Medical Sciences, data pertaining to all patients presenting with congenital lung mass during the 11-year period was obtained from the hospital medical records department. Patients' surgeries were performed by board-certified pediatric surgeons, via the trans-thoracic approach. Imaging studies, including chest radiography, ultrasonography, and CT scans were all performed at Mofid children's hospital, with the exception of prenatal ultrasonographies and fetal MRIs.

The following data was retrospectively studied, based on the medical records: sex, gestational age, age at surgery, patient's first presenting symptom, length of hospitalization, and accompanying features. The prenatal ultrasonographic diagnosis, postnatal radiographic diagnosis, and pathologic diagnosis of each patient was identified, if available.

\section{Results}

Between the years 2004 and 2015, 47 cases were definitely diagnosed as congenital lung mass in Mofid Children's Hospital. Cases of pulmonary cystic lesions were separately analyzed in this study. Of these 37,28 cases (75\%) were boys, and $9(25 \%)$ were girls. The mean hospital stay of the patients was 13.3 days (SD: 10.21). Thirty-six (97.2\%) cases presented with pure pulmonary signs and symptoms, whereas only one $(2.8 \%)$ case was accidentally diagnosed and one presented with non-respiratory features (e.g., fever, vomiting, restlessness, and poor weight gain). Cases were subdivided into three distinct age groups, as follows: group 1 included cases with the age of 28 days and younger; group 2 consisted of patients aged 29 days to 24 months; group 3 was composed of cases with a patient age two years and older. The frequency of cases in each age group was 16 (43.2\%), 17 (46\%), and 4 (10.8\%), respectively.

In terms of prenatal evaluations, unfortunately, 27 (73\%) patients missed the opportunity for early diagnosis. Most of the cases without prenatal diagnosis were later found to be CLE. Ten (27\%) pathologically diagnosed patients had the same radiologic diagnosis.

Of 37 cases, 16 (43.5\%), 12 (32.5\%), 7 (19\%), $1(2.5 \%)$, and 1 (2.5\%) were diagnosed as CLE, CPAM, pulmonary sequestration, hybrid lesion, or bronchogenic cyst, respectively. Lesions were mostly located in the left side of the tho$\operatorname{rax}(21$, or $57 \%$ ) versus the right side (16, or $43 \%)$. Echocardiography was routinely done during the hospital course, but the GI contrast study for detecting any possible gastrointestinal communication was performed in none of the cases. Accompanying features, with the exception of icterus as a common neonatal complaint and pneumothorax and pleural effusion as frequent complications of tho- 
racotomy, were considered as follows: three cases with cardiovascular morbidities (patent ductus arteriosus and atrial septal defect), two cases with hematologic accompanying feature (thrombocytopenia), two cases with central nervous system abnormality (leukomalacia and brain cyst), and one case with hypospadiasis. Of these nine accompanying co-morbidities, four were seen with pulmonary sequestration, three were seen in CCAM, and two were among the CLE cases.

\section{Discussion}

In a study conducted by Langston in Texas children's hospital, 42 cases had the same pathologies as our study. In contrast to the frequency of our cases, their cases included pulmonary sequestration (40\%), CLE (24\%), CCAM (21\%), and bronchogenic cyst (15\%) (5). In the Langston study, male predominance was apparent in all the masses except for CLE, in which the sexes had the same frequency. However, in our study, the male-to-female ratio of CLE cases was 13 to $1(5)$.

In another study in Saudi Arabia, of 57 cases, 65\% were CLE, $12 \%$ were CCAM, $14 \%$ were bronchogenic cyst, and 9\% were sequestration. The prevalence of cystic lesions in the Saudi Arabia study was somewhat comparable to ours regarding the high prevalence of CLE cases (6). Most of our cases with undefined prenatal diagnosis were postnatally diagnosed as CLE (55\%). Although missing some of these screening prenatal diagnoses is inevitable, even in expert hands, paying attention to controversial clues and ambiguous hints should prompt radiologists to apply further modalities, such as ultra-fast magnetic resonance imaging $(7,8)$. Unfortunately, all of the pathology reports neglected to include Immune-Histo-Chemical staining, which is necessary for prediction of probable future malignant transformation $(9,10)$.

CCAM sub-typing in our study was as follows: five cases were type 1 , which is considered the most common and has the best prognosis; two cases were classified as type 2 (one of them was the only hybrid lesion); one case was type 3 and, unfortunately, five cases did not specify typing (1-3). In the study of Adzick et al., excluding type 0, which is incompatible with life (less than $2 \%$ ), most of the CCAM cases belonged to the type 1 category (60\% - 70\%), whereas the least common cases were included in the type 3 group (10). Classification of CCAM lesions into macro-cystic and micro-cystic was not mentioned in our pathologies.

Male gender was significantly (five times) more prevalent in these lesions. In our survey, three cases were diagnosed as ELS, two BPS were not defined regarding their subcategory, one case was a hybrid of ELS and CPAM (type 2), and only one case was an ILS. In the study of Langston in
Texas, ELSs were almost two times more frequent than ILSs, whereas in the study conducted in Saudi Arabia, such classification was not mentioned $(5,6)$. These lesions equally involved the two hemi-thoraces. None of the PSs had the accompaniment of polyhydramnios, hydrops, or diaphragmatic hernia. In spite of the significance of vascular drainage of such lesions, the vascularity of the only hybrid lesion was specified in the surgery notes (i.e., vein drained to azygos and artery derived from the descending aorta).

In our investigation, among 16 cases of CLEs, only one case had prenatal diagnosis; 13 cases were males, and 11 cases were located in the left upper lobe. In this study, none of the CLE cases was diagnosed to have congenital heart disease, although that is expected in $20 \%$ of cases, according to the previous studies $(2,3)$. Polyhydramnios, mediastinal shift, bilateral lesions, existence of hydrops, and larger cysts have been proposed as poor prognostic features (1-3, 10-12).

Our only case of right BC was a six-day old male with associated findings of prenatal polyhydramnios, congenital diaphragmatic hernia, and jejunal atresia. Although he could have benefited from fetal interventions, such as simple amniotic fluid centesis to alleviate polyhydramnios, open fetal surgical resection, thoracoamniotic shunt placement, and percutaneous laser ablation, surgeons decided to postnatally resect the lesions through lobectomy $(13,14)$.

In our study, polyhydramnios was documented in three cases: in a case of CCAM (type 1), in a newborn with bronchogenic cyst, and in a patient diagnosed with congenital lobar emphysema. We found just one bilateral case of CCAM. She was a three-day old female, who underwent bilateral segmentectomy and was discharged in good condition after 25 days of hospitalization. Hydrops was not indicated in any cases. History of prematurity was present in three cases (two CCAM and one pulmonary sequestration). The cases with the oldest patients included an 8-year-old female with sequestration, two 5-year-old females with sequestration, and a 3.5-year-old female with CLE.

\subsection{Conclusion}

Regarding the diversity and significance of congenital pulmonary masses detected in children, in this study, we aimed to identify the characteristics of cystic lesions during an 11-year period in a referral children's hospital in Tehran, Iran. Male predominance was obvious among the cases, as had been found in previous studies.

\section{Acknowledgments}

We would like to express our gratitude to the pediatric surgeons of Mofid children's hospital in Tehran, Iran for 
Table 1. Distribution and Characteristics of Congenital Pulmonary Lesions

\begin{tabular}{|c|c|c|c|c|c|c|c|c|}
\hline Lesion & No. & Right & Left & Bilateral & Age Range & Median Gestational Age, Week & M:F & Prenatal Diagnosis + - \\
\hline CCAM & 12 & 5 & 6 & 1 & 1-124 days & 37.6 & $10: 2$ & $4 / 8$ \\
\hline Pulmonary Sequestration & 7 & 4 & 3 & 0 & 19 days -8 years & 37.4 & $4: 3$ & $3 / 4$ \\
\hline CLE & 16 & 5 & 11 & 0 & 6 days -5 years & 39.8 & $13: 3$ & $1 / 15$ \\
\hline Hybrid lesion $^{a}$ & 1 & 0 & 1 & 0 & 3 days & 38 & $0: 1$ & 1 \\
\hline Bronchogenic Cyst & 1 & 1 & 0 & 0 & 6 days & 38 & 1: 0 & 1 (wrong diagnosis) \\
\hline Total & 37 & 15 & 21 & 1 & 1 day - 8 years & 39.5 & 28: 9 & $10 / 27$ \\
\hline
\end{tabular}

${ }^{\mathrm{a}}$ Hybrid lesion refers to the simultaneous presence of CPAM and sequestration in an individual pulmonary lesion.

their perfect surgeries.

\section{References}

1. Wammer KH, Anderson KC, Erickson PR, Kliegman S, Moffatt ME, Berg SM, et al. Environmental Photochemistry of Altrenogest: Photoisomerization to a Bioactive Product with Increased Environmental Persistence via Reversible Photohydration. Environ Sci Technol. 2016;50(14):7480-8. doi:10.1021/acs.est.6b02608. [PubMed: 27356268].

2. Wilmott JS, Scolyer RA, Long GV, Hersey P. Combined targeted therapy and immunotherapy in the treatment of advanced melanoma. Oncoimmunology. 2012;1(6):997-9. doi: 10.4161/onci.19865. [PubMed: 23189245].

3. Bernard AW, Martin DR, Moseley MG, Kman NE, Khandelwal S, Carpenter D, et al. The Impact of Medical Student Participation in Emergency Medicine Patient Care on Departmental Press Ganey Scores. West J Emerg Med. 2015;16(6):830-8. doi: 10.5811/westjem.2015.9.27321. [PubMed: 26594274].

4. Gornall AS, Budd JL, Draper ES, Konje JC, Kurinczuk JJ. Congenital cystic adenomatoid malformation: accuracy of prenatal diagnosis, prevalence and outcome in a general population. Prenat Diagn. 2003;23(12):997-1002. doi:10.1002/pd.739. [PubMed:14663837].

5. Langston C. New concepts in the pathology of congenital lung malformations. Semin Pediatr Surg. 2003;12(1):17-37. doi: 10.1053/spsu.2003.00001. [PubMed: 12520470].

6. Al-Salem AH, Matta H. Congenital Cystic Diseases of the Lung in Infant and Children: Our Experience with 64 Cases. J ped surg special. 2015;9(2):1-52.
7. Williams HJ, Johnson KJ. Imaging of congenital cystic lung lesions. Paediatr Respir Rev. 2002;3(2):120-7. [PubMed: 12297058].

8. Wall J, Coates A. Prenatal imaging and postnatal presentation, diagnosis and management of congenital lung malformations. Curr Opin Pediatr. 2014;26(3):315-9. doi:10.1097/MOP.0000000000000091. [PubMed: 24739492].

9. Roggin KK, Breuer CK, Carr SR, Hansen K, Kurkchubasche AG, Wesselhoeft CJ, et al. The unpredictable character of congenital cystic lung lesions. J Pediatr Surg. 2000;35(5):801-5. doi: 10.1053/jpsu.2000.6088. [PubMed: 10813354].

10. Scott Adzick N, Harrison MR, Glick PL, Golbus MS, Anderson RL, Mahony BS, et al. Fetal cystic adenomatoid malformation: Prenatal diagnosis and natural history. J Pedi Sur. 1985;20(5):483-8. doi: 10.1016/s0022-3468(85)80470-x.

11. Bunduki V, Ruano R, da Silva MM, Miguelez J, Miyadahira S, Maksoud JG, et al. Prognostic factors associated with congenital cystic adenomatoid malformation of the lung. Prenat Diagn. 2000;20(6):459-64. [PubMed: 10861709].

12. Bianchi D, Crombleholme T, D'Alton M, Malone FF. Diagnosis and Management of the Fetal Patient. 2 ed. UK: McGraw Hill; 2010. pp. 3415.

13. Knox EM, Kilby MD, Martin WL, Khan KS. In-utero pulmonary drainage in the management of primary hydrothorax and congenital cystic lung lesion: a systematic review. Ultrasound Obstet Gynecol. 2006;28(5):726-34. doi:10.1002/uog.3812. [PubMed: 17001747].

14. Merchant AM, Peranteau W, Wilson RD, Johnson MP, Bebbington MW, Hedrick HL, et al. Postnatal chest wall deformities after fetal thoracoamniotic shunting for congenital cystic adenomatoid malformation. Fetal Diagn Ther. 2007;22(6):435-9. doi: 10.1159/000106350. [PubMed: 17652932]. 